\title{
Evaluative tools in impact investing: Three case studies on the use of theories of change
}

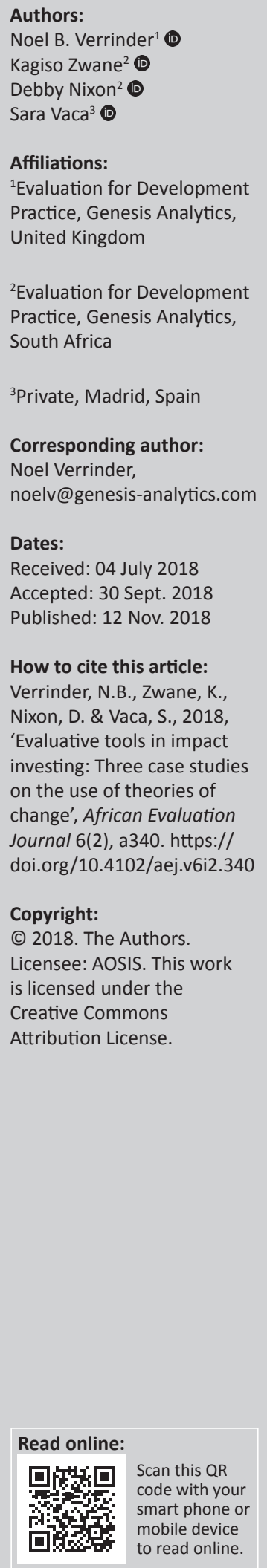

Authors:

Bel Verrinder

Debby Nixon ${ }^{2}$ (D)

Sara Vaca $^{3}$

Affiliations:

Practice, Genesis Analytics,

Practice, Genesis Analytics,

${ }^{3}$ Private, Madrid, Spain

Corresponding author:

Noel Verrinder,

Dates:

Accepted: 30 Sept. 2018

How to cite this article:

Verrinder, N.B., Zwane, K

investing: Three case studies

of the use of theories of

Journal 6(2), a340. https://

doi.org/10.4102/aej.v6i2.340

Copyright:

(1)

to read online.
Impact investing is becoming one of the largest forces in driving social and environmental change globally. However, how one defines, measures and communicates this impact is not well defined or consistently implemented. This can prevent investors from making wellinformed decisions and allows for 'impact washing'. The evaluation community has many tools that could be adapted and used in the world of impact investing. Theories of change allow for the better communication of impact, identification of indicators to be measured and critical interrogation of logic. The attributes of theories of change could assist in steering the growing force of impact investing towards gathering more investment and achieving greater impact. This paper is exploratory and examines the development and use of theories of change as a tool for impact investing and seeks to identify the benefits of the tool. We qualitatively review three case studies of organisations that have implemented theories of change and identify common key themes. We find that theories of change are a useful tool for the communication of impact, identification of indicators to be measured and for the critical interrogation of logic. However, theories of change do not provide a panacea to the impact challenge; the need to rigorously measure impact is not fulfilled by merely identifying what needs to be measured. Regardless, the use of theories of change adds an advantage in a space where others have not gone to the same length to show their commitment to driving change.

\section{Introduction}

Impact investing is becoming one of the largest forces driving social and environmental change globally. However, how one defines, measures and communicates impact is not well defined or consistently implemented. Not being able to assess and communicate impact prevents investors from adapting their investment strategies to maximise impact or generate sufficient interest in truly impactful investments. Conversely, a lack of consistency and rigour in impact measurement may allow investors to claim impact that may not exist or over exaggerate the impact of their investment, which has been called 'impact washing'.

The evaluation community has many tools that could be adapted and used in the world of impact investing to overcome these issues. Theories of change allow for the better communication of impact, identification of indicators to be measured and critical interrogation of logic. The attributes of theories of change could assist in steering the growing force of impact investing towards gathering more investment, achieving greater impact and avoiding the occurrence of 'impact washing'.

This paper investigates the development and use of theories of change in capturing the logic behind the various three organisations in the impact investing space and evaluates how the use of theories of change have benefited these organisations and their investments.

\section{Impact investing}

Impact investing has generated significant interest over the past decade as evidenced by the growth of impact investors, market intermediaries and measurement frameworks amongst others (Ebrahim \& Rangan 2014; Picciotto 2011). The common theme across impact investing is creating positive social change alongside financial returns with impact investors being differentiated by their choice of sector or funding instruments. Indeed, what separates impact investments from earlier forms of socially aligned or socially responsible investments is the intention behind the investment to have a positive effect on society as opposed to avoiding negative effects (Flynn, Young \& Barnett 2015). This intentionality is reflected in the working definitions of impact that have been adopted by several prominent industry players and pioneers such as the Global Impact 
Investing Network (GIIN) that defines impact investments as those that: 'aim to solve social or environmental challenges while generating financial profit'. Similarly, the UK-based investment firm Bridges Ventures defines impact investing as: 'actively placing capital in businesses and funds that generate social and/or environmental good and a range of returns, from principal to above market, to the investor' (Flynn et al. 2015, O’Flynn \& Barnett 2017).

By having a deliberate focus on achieving positive social or environmental outcomes, impact investment necessarily calls for measuring social and environmental returns alongside financial returns and potentially weighing them up against one another. This has given rise to a multitude of tools for and approaches to measuring impact across the sector (O'Flynn \& Barnett 2017). Perhaps the most prominent of these is the Impact Reporting and Investment Standards (IRIS) conceptualised by the GIIN. Others include the Acumen Fund's Lean Data methodology, Social Return on Investment (SROI) and the Global Reporting Initiative (GRI) which assess Environmental, Social and Governance (ESG) performance (O'Flynn \& Barnett 2017).

Alongside these industry standards, there is a portion of the literature that presents independently developed approaches to social impact assessment. Finally, in an effort to standardise the impact measurement, the Social Impact Investment Taskforce (2014) has produced a subject paper on measuring impact. This paper outlines seven best practice guidelines that impact investors can integrate into their investment management: Set Goals; Develop Framework and Select Metrics; Collect \& Store Data; Validate Data; Analyse Data; Report Data; and Make Data-Driven Investment Management Decisions.

In addition to the tools and methods focused literature, there is also a body of knowledge that seeks to advance the debate around impact measurement in a theoretical sense. There is an acknowledgement in the literature that standardised metrics often fall short of measuring outcomes, which calls for the adoption of multiple methods in evaluating the impact of an investment (Brest \& Born 2013). There is a broad range of data collection and analysis methods that can be adopted to meet this. There is a growing consensus that the most relevant and immediately translatable evaluative tool for measurement in impact investing is the theory of change (Flynn et al. 2015; Jackson 2013; Jackson \& Harji 2014).

\section{Theories of change}

In programme evaluation, the theory of change is used to identify and test causal mechanisms of an investment and the assumptions made in the path from activities to desired impacts. It can also be used to inform the improvement of strategies and implementation plans. Importantly, a theory of change must include (usually depicted graphically) the underlying logic, assumptions, influences, causal linkages and expected outcomes of a development programme (Jackson 2013; W.K. Kellogg Foundation 2004).
Impact investors can use theories of change at various points in the investment process. Firstly, in performing due diligence when selecting investments, the theory of change can act as a framework to communicate with an entrepreneur or investee about their intended impact, underlying assumptions and potential strategies for achieving their intended impact (So \& Staskevicius 2015). Importantly, investors can use the theory of change to identify areas where assumptions and causal pathways are untested or unproven with a view to testing the overall business model (So \& Staskevicius 2015).

Theories of change can also be used to determine what investors should measure and to set impact targets for the investees (So \& Staskevicius 2015). As the theory of change explicitly shows the path from resources to outcomes, investors and entrepreneurs can use it to determine the key performance indicators (KPIs) for outcomes and outputs and that must be monitored as part of the impact management and measurement process. Prior to setting specific indicators, an investor may also use the theory of change to determine the level that they are interested in measuring. Linked to selecting indicators is the use of the theory of change to create incentive systems based on social impact such as funding milestones for the enterprise or performance bonuses for the managers (So \& Staskevicius 2015).

Finally, by involving funders, investees and practitioners, impact investors can use their theories of change to communicate their impact in a way that aligns the stakeholder needs in a cohesive message. The theory of change is often graphically depicted in a simple way that resonates with audiences that may not be familiar with impact measurement approaches. This is particularly useful when an entrepreneur or impact investment fund is trying to raise funds from financial institutions.

To draw conclusions on the value add and shortcomings of theories of change, this paper explores and describes how three organisations have used theories of change to articulate their impact logic, communicate their purpose and measure their outcomes..

\section{Research methods and design}

The three cases selected for this paper are of organisations that use theories of change to describe the impact they are aiming to achieve and how their activities contribute to this impact. The organisations were selected as case studies for the following reasons:

- The organisations have adopted theories of change.

- They are active on the African continent.

- They are involved or associated with impact investments.

- The diversity of the industries they operate in.

The authors are familiar with the three cases, as the organisations are previous clients of Genesis Analytics.

For each case, we present the aims and activities of the organisations selected to be case studies to provide the reader 
with context. After this, we discuss the original reasons as to why the organisations decided to explore the use of theories of change and the challenge of their development. We then walk through the process of development to provide insight into the requirements and practicalities of building a theory of change, which is followed by a brief description of the output. Finally, we unpack the benefits of using theories of change.

After the presentation of the cases, we draw out similarities to establish key themes and discuss the relevance and usefulness of using theories of change.

\section{Results}

\section{Case 1: TUHF (formerly the trust for Urban Housing Finance) \\ Background}

TUHF was founded in 2003 as a commercial property finance company with a vision to provide effective, cost-efficient solutions to inner-city improvements in South Africa. TUHF works with asset managers, commercial banks and development finance institutions to bring about inner-city rejuvenation and sustainable economic empowerment. A portion of the building stock in the urban centres of South African cities have not been maintained, updated or kept to basic safety standards and are often home to many people who are at risk of fires, building collapse and crime. TUHF's investments are aimed at developing affordable, high-quality housing stock in urban centres, with a vision of creating vibrant, safe and mixed-income living spaces in the centre of South African cities.

Through careful assessment of inner-city areas within South Africa, TUHF compiles a predefined list of high potential areas for possible financing exhibiting signs of urban decline. TUHF then identifies potential entrepreneurs that are interested in property upgrades, refurbishments, extensions, conversions, redevelopment and new developments of properties in the inner city.

Once both a potential property and a potential entrepreneur have been identified, TUHF will provide financing to the qualifying entrepreneur. A value-addition that sets TUHF apart from traditional finance providers is that the entrepreneurs are provided with a property entrepreneurship training course. After the entrepreneurs have secured finance from TUHF, they proceed to upgrade their building(s). To do this, an entrepreneur will employ a construction company or sub-contractor for the required trade and once the building has been upgraded, they will employ a property management solution to ensure that the property is well-run and maintained.

Through this process, affordable high-quality housing stock is made available in inner cities for rental to individuals and families. Individuals or families will in turn pay rent and utilities, contributing to the local government's fiscus, to lease the units and, slowly but surely, there is an increase in affordable quality residential rental stock available in innercity areas. Through increasing the number of people in the targeted areas, businesses develop to meet the needs of these residents and thus contribute to local economic development.

\section{The challenge}

TUHF's core business is providing finance to emerging property entrepreneurs whose business is in refurbishing neglected, under-maintained and dilapidated buildings in central urban areas. Supporting such enterprises, which struggle to source traditional affordable financing, contributes to creating sustainable inclusive economic development and also provides financial returns.

While the impact resulting from the finance that TUHF provides to enterprises it directly funds was easy to understand and measure through standard business monitoring activities, measuring the broader impacts on employment, local fisci, crime, communities and the environment required a deeper understanding of the logical chain of events that lead to their achievement. Only once this logic was captured and understood could these outcomes be identified, defined and measured.

There was internal acceptance that TUHF had positive impacts on the entrepreneurs it targeted, as well as the communities and inner cities in which they operated. However, there was still a need to provide evidence of this, and that is why a comprehensive impact measurement framework that could guide the measurement of results and capture lessons, to inform better management, was sought by TUHF.

\section{The process}

TUHF embarked on a journey to critically assess its systems and develop a framework and tools to improve on its data collection, which would allow for better impact reporting in the future. The development of such a framework required an easy to understand, but comprehensive, expression of how TUHF was facilitating enterprise development and contributing to broader impacts. To complete this task, champions were selected within TUHF to own the process and the consultant was contracted to facilitate and to guide the process using international standards, such as the Donor Committee for Enterprise Development (DCED) Standard.

The process of creating this framework hinged on the development of a theory of change for the organisation. The articulation of the theory of change occurred towards the end of 2015 and early 2016 and was completed through an iterative and consultative process. The process was completed by engaging with TUHF stakeholders and asking questions about TUHF's investment protocol, philosophy and anticipated impacts, as well as reporting processes and requirements. Numerous engagements were conducted with both internal 
and external stakeholders to TUHF. Key to this process was engaging with members from various levels of the organisation, including the visionaries within the organisations, such as the chief operating officer (CEO), to the data managers in the IT department, to those who would be collecting data, such as the loan officers. This allowed for the synthesis of a holistic view of what the organisation contributes to, and what would be possible to measure.

The iterative and consultative process was essential to create ownership and commitment to the use of the framework within TUHF, taking into consideration the capacity and feasibility of framework tasks. This was necessary to enable the level of engagement and rigour required for long-term, widespread, sustainable implementation and subsequent results and impact measurement. To achieve this level of engagement, the TUHF identified a 'champion' to work with the consultants. The champion worked closely with the consultants, had regular meetings, and the consultant worked from the TUHF offices from time to time.

\section{The output}

The theory of change started by taking the form of a results chain; however, this did not entirely resonate within the organisation, and through the process of iteration the theory of change took on a unique structure, and consensus on the anticipated outcomes and impacts of the organisation was reached. There was a further process of wordsmithing to develop a unified language of impact that would resonate internally and externally. The final output was a diagram explaining how the activities of TUHF linked to the outcomes and eventual impacts it was aiming to achieve, along with a set of indicators to measure progress to this end. Although not a results chain, the bespoke illustration still met the important criteria of explaining how impact is achieved and could still be used to identify indicators for measurement.

\section{Outcome}

At the time of drafting this paper, 2 years had passed since the finalisation of the theory of change for TUHF. Since adopting the theory of change and associated framework, TUHF has experienced the following benefits:

- TUHF has been able to communicate its impact story more consistently with its investors and hence has been able to harmonise how it reports on impact. This is owing to a shared understanding of impact and more consistent measurement internally.

- Being able to better communicate its impact story has helped TUHF secure funding from less traditional sources, such as grant funding from government and debt from development finance institutions. TUHF reports that investors are surprised and impressed that they have a theory of change and measurement framework. This makes them stand out in their field.
- The theory of change means that testing of impact logic is now embedded in all new product trials, which has assisted TUHF launch new innovative products with a greater impact focus.

Nevertheless, some challenges do exist, such as how to retrofit older products to include the framework and how to ensure consistent measurement. To overcome the last point, TUHF has made certain indicators, gleaned from the theory of change, part of staff KPIs. This has helped further change the culture of the organisation to be more impact measurement and impact focused; however, this is not a direct result of the use of a theory of change.

\section{Case 2: The technical assistance facility to the African Agricultural Fund}

\section{Background}

The African Agriculture Fund (AAF) is a private equity fund, established in 2009, whose investments seek to achieve positive impact on African agriculture and food production. African Agriculture Fund established the Technical Assistance Facility (TAF) in 2011 to assist the medium-term economic development of small and medium enterprises (SMEs) and small-scale farmers supported by the AAF. Technical Assistance Facility is implemented by TechnoServe Inc. and focuses on enabling SMEs, small-scale farmers, farmers' organisations and cooperatives to benefit, either directly or indirectly, from investment windows of the AAF. The TAF does this by:

- Strengthening the linkages between small-scale farmers, farmers' organisations, cooperatives and AAF investments.

- Strengthening the capacities of SMEs so that they can more readily secure financing through the AAF.

- Supporting coordinated, commercial relations based upon mutual trust and a long-term business interest for successful outgrower schemes in the agricultural supply chain.

- Increasing the ability of small-scale farmers and SMEs to receive debt financing through rural financing sources.

Through these activities, TAF seeks to build on the AAF financial investment to support a larger impact objective improved food security in Africa. The TAF has implemented projects in 12 African countries.

\section{The challenge}

In 2014, the TAF determined that its monitoring and evaluation (M\&E) system, while internally logical, consistent with the TAF Logical Framework structure, could be improved by articulating how it contributes to food security in a more consistent and explicit manner. A specific area to be improved was providing the system with the ability to explain how each project contributed to portfolio-level impact.

Along with this more explicit explanation of how each TAF project contributed to food security, the TAF wanted to know 
what to measure to ensure that its projects were on track to achieve better outcomes for its beneficiaries. Thus, the TAF commissioned an evaluation and redesign of their M\&E system.

It was important to be cognisant of the fact that those supported by TAF run on a for-profit basis and, thus, the reporting and measuring standards had to be realistic and not too onerous and were also able to capture the necessary data for comprehensive evaluation.

\section{The process}

The approach to revising the M\&E system was guided by an understanding of market systems, expertise in agribusiness and knowledge and experience applying international M\&E standards in practice.

The process began with a review of good practices to produce a practical system that was useable and aligned to recognised international standards. The DCED Standard was used as a starting point, as it provides a practical framework for programmes to monitor their progress towards their objectives, enabling them to better measure, manage and demonstrate results. In particular, the DCED Standard was developed to build the M\&E frameworks for interventions addressing systemic change for enterprise development. What is core to the DCED Standard is the use of results chains as a depiction of the theories of change of the interventions and this is what made it attractive for this process.

Next, stakeholders were consulted to understand each organisation's needs and capacities; the primary stakeholders included the European Commission (EC), International Fund for Agricultural Development (IFAD), the AAF fund managers, the TAF implanting agency and representatives from benefiting organisations. This provided the insight required to build the theory of change and also generate buyin and capacity.

\section{The output}

The process culminated in an overall results chain for the TAF and a template intervention-level results chain that covered the potential interventions the TAF may undertake. Figure 1 illustrates how the intervention-level results chains nested in the facility level.

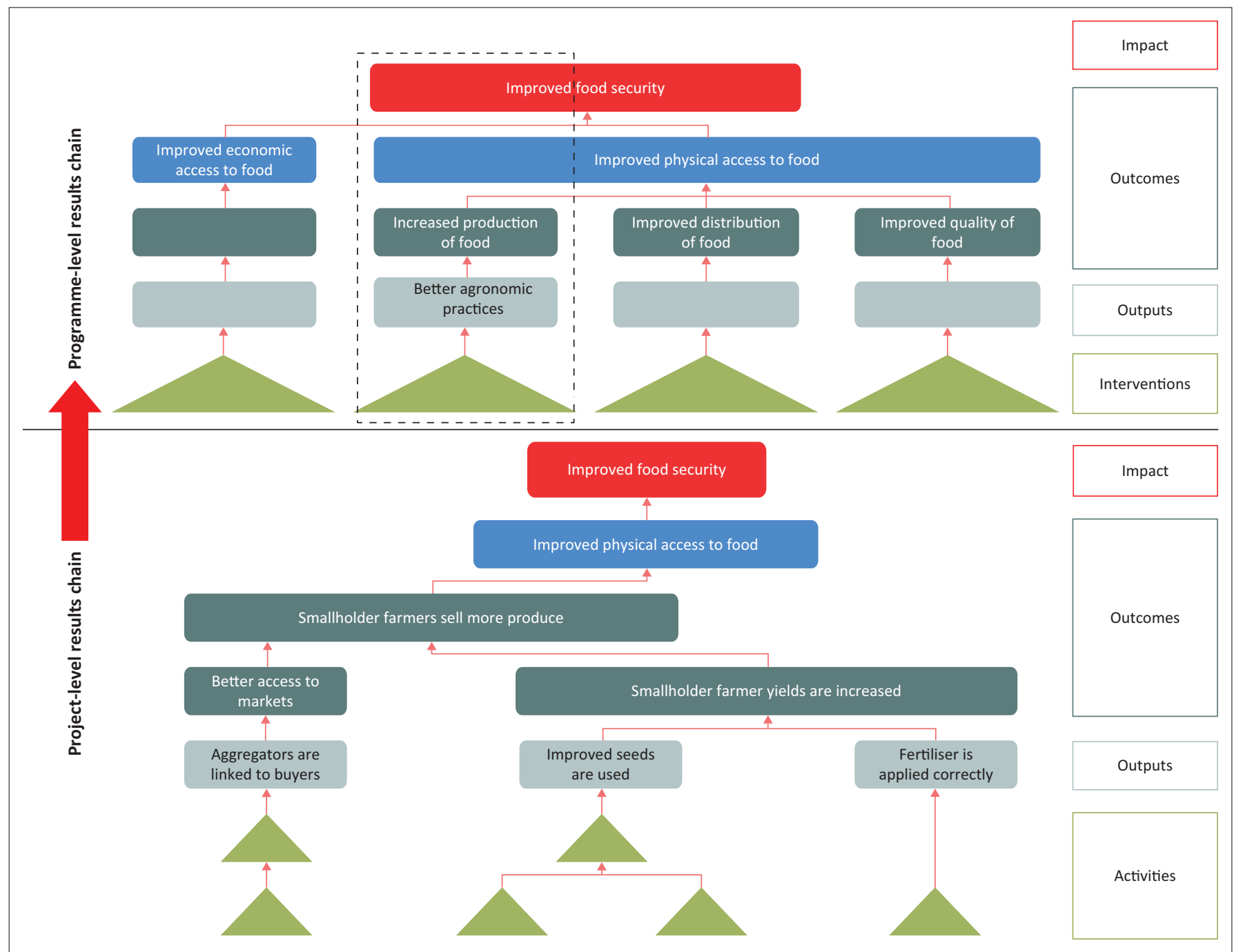

FIGURE 1: Stylised results chains for Case 2 (content removed). 
This approach allowed the TAF to identify key outcomes that all of its projects should contribute to that feed into food security. Specifically, these included outcomes around economic and physical access to food.

\section{Outcome}

The TAF has been using its theory of change and measurement framework since 2014. The key benefits that have flowed from using theories of change have been primarily experienced during the design phase of new projects. Developing the detailed results chains for each technical assistance grant during its design has encouraged the rigorous interrogation of assumptions, as well as the identification of additional activities to fill gaps and ensure greater contribution to impact.

Having better physical and economic access to food as nonnegotiable constants in the results chains of projects has also allowed TAF to consistently illustrate how it contributes to food security across its various intervention types. This assists in communicating the purpose of each project, which then helps with generating buy-in from stakeholders and investors.

The TAF relies on third-party technical assistance providers to deliver its interventions. These service providers often lack the specific skills needed to build a theory of change or measure their results. This has presented a challenge in ensuring consistency of measurement across their portfolio of investments. This challenge is not unique to TAF; many programmes that rely on externals to implement their projects can struggle to find contractors with both strong technical implementation capacity and strong M\&E expertise. Thus, to overcome this, the TAF allows these implementers to first articulate their intervention logic in their own way, which is not necessarily the same as the results chain method that the TAF uses. The TAF then works with the implementor to crystallise this intervention logic into its standard results chain form, which allows them to have a unified language for describing their impact, while also building the capacity of the implementor to develop theories of change in the future. In addition to this individual-level training, the TAF has held M\&E capacity-building sessions for its technical assistance service providers.

Nevertheless, while the theories of change have helped with identifying what to measure, the service providers still struggle with measuring the intermediate and high-level outcomes, such as job creation and income improvements because, not only do these take time to be realised, but they are also expensive and difficult to get data on. In instances where the measurement is not possible or too resourceintensive, the TAF uses theory, multipliers and research to establish proxies for these indicators.

\section{Case 3: A fund focused on developing capital markets in Africa}

\section{Background}

A development finance institution (DFI) established a fund (the Fund) in 2012 to promote the use of new debt instruments as a viable source of funding for firms in Africa. The Fund focuses on working with organisations and companies that operate in renewable energy, housing, health, education, infrastructure, agriculture, microfinance and wholesale refinancing. The organisations it supports and invests in target low-income households and micro, small and medium enterprises (MSMEs).

The vision for the Fund emerged from a G20 summit linked to underdeveloped capital markets in Africa. Capital markets are necessary to improve the systemic well-being of African economies through their local currencies. The Fund's aim is to catalyse investment through driving the use of innovative debt instruments issued by local businesses, as well as acting as an anchor investor to encourage crowding in of other investors. The Fund also has a TAF, which it uses to engage in investee capacity building. Launching innovative debt instruments requires a great deal of assistance, and these initial costs are often covered by the TAF to ensure buy-in for the instruments. Beyond financial assistance, the TAF supports the business in managing their debt instruments and focusing their strategies to better achieve impact.

Through its investments and activities, the Fund improves the sustainability and diversity of funding sources for the businesses it works with, while reducing systemic risk across the financial sector and driving capital market development.

\section{The challenge}

The Fund has had a clear developmental mandate that includes improving the capacity of local currency capital markets in Africa through innovative debt instruments issued by companies operating in developmental sectors. Although the Fund had an implied theory of change alongside specific social and environmental impact indicators, following the advent of the Sustainable Development Goals (SDGs), there arose a need to formalise its theory of change framework to better define and communicate how its current activities are achieving its mission and contributing to these goals.

There was also a need for the theory of change to allow the Fund to connect its activities in capital market development to benefits for MSMEs and enable the Fund's team to communicate their developmental purpose and logic in a unified language they could use with its investors, investees and other stakeholders alike.

Unlike the other two cases, defining a unified impact goal for this Fund's investments presented a challenge, as the Fund does not operate within one sector of an economy. Although its underlying focus is on developing local capital markets, it works with and invests with organisations from a variety of industries and sectors, making its pathway to impact somewhat investment dependent.

However, the one unifying factor that simplifies this is that the Fund's primary mechanisms of driving change within the 
organisations it supports are similar across its investments. That is, the Fund assists organisations raise capital for their operations through supporting them launch innovative debt mechanisms, provides anchor investment if needed and assists in addressing market-level constraints.

\section{The process and output}

Given the focus on linking the Fund to the SDGs, the first step of this process involved engaging with the Fund's stakeholders and investees to generate consensus of which of the SDGs the Fund contributed to. Three SDGs were identified as being ubiquitous across the Fund's investments, those being Good Jobs and Economic Growth (SDG 8), Partnership for the Goals (SDG 17) and a culmination in No Poverty (SDG 1). Climate Action (SDG 13) was found to also be an important SDG across a variety of the areas the Fund invests in. Sector-specific SDGs that are dependent on the industries the investees operate in were also identified. These covered an additional eight of the SDGs, for example, No Hunger (SDG 2) for investments in the agricultural sector, Good Health (SDG 3) for investments in organisations focusing in the health sector and Clean Energy (SDG 7) for investments in renewables.
The activities, outputs and outcomes of the Fund were mapped to the identified SDGs, and through an iterative process an adapted results chain model was used to articulate the theory of change (see Figure 2). The results or boxes were then categorised and overlaid on top of theme areas. The pathway for how this commercial interest would be achieved was embedded in the primary chain.

\section{Outcome}

At the time of drafting this report, the theory of change was still in draft form and the process of refining the framework ongoing. Nevertheless, the process of developing the theory of change has generated outcomes itself. Specifically, the process has catalysed discussions between the Fund's management, the board and investors and moved the topic of 'impact' to centre stage. This is important as it is part of a longer evolution of moving from a focus of investments meeting certain investment criteria to thinking through what the investment is actually aiming to achieve, if the thought behind it is plausible and whether it is likely to achieve impact.

However, the challenge of measurement will still need to be overcome. While the theory of change process has allowed

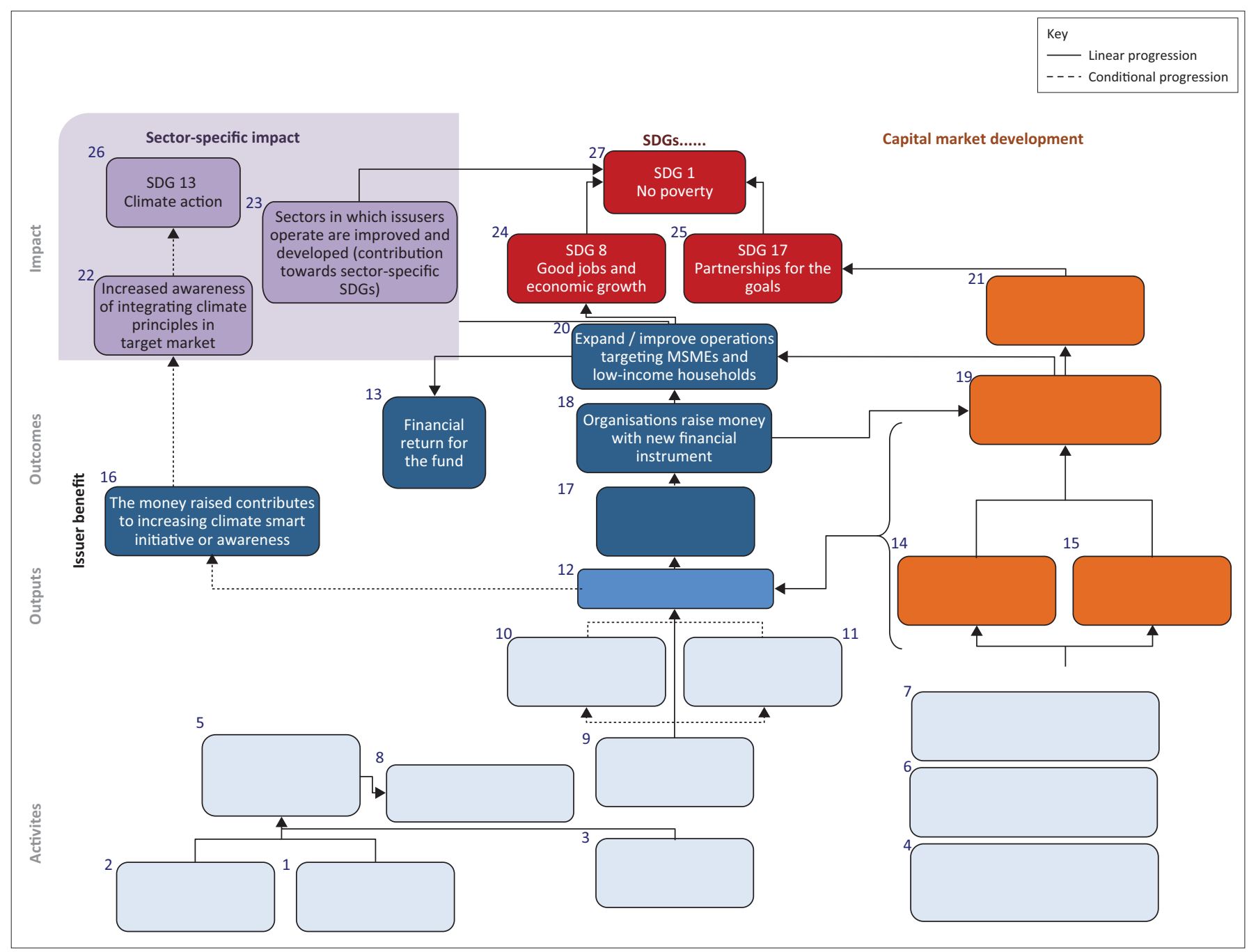

SDG, sustainable development goals; MSMEs, micro, small and medium enterprises.

FIGURE 2: Stylised theory of change for Case 3 (content removed). 
for the identification of key indicators, the measurement of these can require additional resources. A question ubiquitous in this space remains on how to measure outcome indicators far removed from the Fund, and how this measurement is resourced and paid for. While it does not solve this problem, the theory of change may be able to alleviate some of the pressure to focus entirely on measurement, as if it is logically sound and backed with extant evidence, the plausibility of the Fund contributing to change is improved and can be communicated.

\section{Discussion}

While the three cases presented in this paper are distinct in contexts, modes and aims, there are similarities between the organisations in terms of what spurred them on to build theories of change. There are three salient commonalities:

- The need to communicate purpose and generate an understanding of the mechanisms behind how impact is achieved. This does not only include the need to communicate with external stakeholders but also the need to build consensus internally and develop an organisational language for articulating impact.

- The need to create a plausible explanation of how the organisation is contributing to changes in outcomes. Although this is more explicit in one of the cases, it is ubiquitous in underlying the first need to communicate.

- The need to identify what to measure to support the provision of evidence of impact. Each case presents the need to show evidence that the organisation is contributing to changing outcomes and this requires the identification of indicators of this change.

While these drivers of why the organisations were required to build theories of change are predominantly fuelled by external funders and stakeholders, benefits flowing from the development process and the subsequent use of the frameworks have also been experienced internally. The benefits identified in these case studies include becoming more attractive to investors, more rigorous in interrogating assumptions and highlighting impact and purpose.

The cases also illustrate that the development and ongoing use of the theories of change are not without challenges and do not provide a panacea to the impact challenge. Because the process requires individuals to input into the process, significant effort is required in on-boarding those brought in after the theory of change has been developed. This effort includes investing time and effort into capacity building. In addition to on-boarding those who were not involved in developing the theories of change, the development process itself can present challenges and requires a concerted effort to reach consensus on and acceptance of the underlying logic of an investment.

Although the final theory of change may often appear simple in each of the cases, to ensure that there is sufficient credibility and buy-in, the process needs to be collaborative, iterative and logically tested repeatedly. This process requires significant time and commitment from all parties; however, the process itself provides important self-reflection and criticism, which results in a stronger belief, internally and externally, in what the organisations are doing. Interestingly, although each case began by exploring using a results chain approach to articulate the theory of change, each organisation adapted the approach in their own way to make the theory of change to internally resonate with staff. Given the focus on communication, the tailoring of the visualisation of the theory of change to be understandable by those who use it is vital and should, therefore, take precedence over compliance with a standard.

While the theories of change and their development are useful in improving credibility and allow for better communication of logic, their development and the identification of indicators alone do not solve the issues around the measurement process. There are additional challenges that are experienced when moving from identifying what to measure to actually measuring, which a theory of change cannot solve in isolation.

\section{Conclusion}

The case studies in this paper have illustrated that theories of change are a useful tool for the communication of impact, identification of indicators to be measured and the critical interrogation of logic, and currently add an advantage in a space where others have not gone to the same length to show their commitment to driving change. While the process to harmonise and standardise how one defines, measures and communicates impact is ongoing, organisations should not shy away from theories of change. Until there is a standard that can be applied, impact investors should articulate their theories of change in their own way, while remaining honest and rigorous, as there are benefits experienced through the development process and implementation of the theory of change. However, theories of change alone do not provide a panacea to the impact challenge and also come with trials in their implementation. Specifically, the need to rigorously measure impact is not fulfilled by merely identifying what needs to be measured, how it will be measured and by whom. Measurement requires its own investment, resources, commitment and research.

\section{Acknowledgements}

We acknowledge the two organisations, TUHF and the TAF to the African Agricultural Fund, which allowed us to use their experiences as case studies in this research paper. Their contribution has been invaluable to this research and we commend their openness.

\section{Competing interests}

The authors declare that they have no financial or personal relationships that may have inappropriately influenced them in writing this article.

\section{Authors' contributions}

N.B.V. wrote the case studies, discussion and conclusion sections of the paper and reviewed the final paper. 
K.Z. conducted the literature review for the paper. D.N. reviewed the literature review section and contributed to discussions and the thinking behind the paper. S.V. provided valuable insights into theories of change and was involved in the thinking and conceptualisation of the article.

\section{References}

Brest, P. \& Born, K., 2013, 'Unpacking the impact in impact investing', Stanford Social Innovation Review, 11(4), 1-14.

Ebrahim, A. \& Rangan, V.K., 2014, 'What impact? A framework for measuring the scale and scope of social performance', California Management Review 56(3), 118-141. https://doi.org/10.1525/cmr.2014.56.3.118

Flynn, J., Young, J. \& Barnett, C., 2015, Impact investments: A literature review, CDI Practice Paper, p. 11, viewed n.d., from www.cdimpact.org
Jackson, E.T., 2013, 'Interrogating the theory of change: Evaluating impact investing where it matters most', Journal of Sustainable Finance \& Investment 3(2), 95-110. https://doi.org/10.1080/20430795.2013.776257

Jackson, E.T. \& Harji, K., 2014, Assessing impact investing: Five doorways for evaluators, The Rockefeller Foundation, New York. Evaluation office, pp. 2-24.

O'Flynn, P. \& Barnett, C., 2017, Evaluation and impact investing: A review of methodologies to assess social impact, viewed n.d., from https://opendocs.ids.ac.uk/opendocs/ bitstream/handle/123456789/12835/ER222 Evaluationandlmpactlnvesting AReviewofMethodologiestoAccessSociallmpact.pdf?sequence=1\&isAllowed=y

Picciotto, R., 2011, Evaluating development philanthropy in a changing world, The Bellagio Initiative, The Rockefeller Foundation, New York.

So, I. \& Staskevicius, A., 2015, Measuring the 'impact' in impact investing, Harvard Business School, Cambridge, MA.

Social Impact Investment Taskforce, 2014, Measuring Impact - Subject Paper Of The Impact Measurement Working Group, viewed n.d., from https://iris.thegiin.org/ research/measuring-impact-subject-paper-of-the-impact-measurement-workinggroup/summary

W.K. Kellogg Foundation, 2004, Using logic models to bring together planning, evaluation, and action: Logic model development guide, W.K. Kellogg Foundation Publisher location, Michigan. 